\title{
The Automagic Box of Beauty A Prototypical Smart Device as Use Case Example for User-Centered Decision Support via the Hub-of-all-Things
}

\author{
Helen Oliver \\ University of Cambridge Computer Laboratory, William Gates Building, 15 JJ Thomson Avenue, Cambridge, U.K. \\ helen.oliver@cl.cam.ac.uk
}

\begin{abstract}
Keywords: Smart Devices, User-Centered Decision Support, Internet of Things, Generativity, Personal Data Stores, Ubiquitous Computing, Sensor Networks, Smart Homes, Usage Context-based Design.

Abstract: In this position paper we present the Automagic Box of Beauty, a prototype smart cabinet which enables the user to track their rate of consumption of toiletries. By transmitting product consumption data from the box to the Hub-of-all-Things (HAT), a platform for personal data that is fully owned and controlled by the individual, we open the potential for decision support for the user that hitherto has only been available at the enterprise level. By returning ownership of personal data to the individual, the HAT enables horizontal integration of information that has until now been held in vertical silos. We show how, by contextualizing the product consumption data with data from a variety of other sensors and sources, the system will support individual users in making decisions - in this use case example, decisions about replenishment and selection of the products in the box.
\end{abstract}

\section{INTRODUCTION}

This article describes the Automagic Box of Beauty (Beautybox), a prototype smart device which demonstrates an example use case for user-centered decision support within the Hub-of-all-Things (HAT) (Ng, 2014).

The HAT is an open platform for personal data stores which are fully owned and controlled by the individual $(\mathrm{Ng}, 2014)$. Developers will find in the HAT an attractive market with much demand for applications to enable user-centered decision support. The HAT will also provide a platform for individuals to market their contextualized personal information to other stakeholders (World Economic Forum, 2013), giving companies unprecedented opportunities to understand the contexts which influence consumer decisions.

While there is an established need for contextual intelligence in the Internet of Things (Vermesan et al., 2014), the HAT will enable users to benefit from unprecedented access to their own personal data and unprecedented opportunities to do their own contextual analysis of their behaviour patterns according to their own ideas about what contextual information is meaningful (Dourish, 2004).
The Beautybox is a smart cabinet which contains everyday toiletries, tracking the products and their rate of consumption, and uploading the data to the HAT platform, the endpoint being a single database instance for a single user. By measuring the individual user's rate of consumption of a product, the system will be able to predict when that product needs to be replenished and enable automation of reordering without user intervention. An application is in progress to provide this functionality, and will also allow the user to specify what action to take if a product is out of stock (list other products to substitute, specify acceptable substitute products by ingredient, and so on).

At this stage of the work in progress, the user is a volunteer known as a Digital Person Zero (DP0) who has consented to donate their personal data to this research effort $(\mathrm{Ng}, 2014)$. The motivation in creating the Beautybox is to make a start in connecting the DP0's home with HAT-enabled devices - beginning with the bathroom, and adding devices until the whole house is covered by a HAT sensor network. By uploading timestamped data from the DP0's entire sensor network as well as their choice of application data and social media data, it is possible to contextualize and make sense of behaviour patterns. For example, imagine that on particular nights, certain lights are switched on and 
are not turned off. Context is provided by looking at the other data from those nights and the mystery is solved: it always happens after watching a horror movie on Netflix.

At the time of writing, two Beautyboxes have been successfully deployed to their respective DP0s and work is in progress to develop a decision support application using the Beautybox data. In this article we will describe the Beautybox, and the application we are in the process of developing for the Beautybox data.

In this section, we have described what the HAT project is, what the Beautybox is, what a DP0 is and the current stage of deployment of the Beautybox, and how we intend to use the data generated by the Beautybox.

In section 2, we will explain the scope of this position paper and what this article is and is not about. In section 3 , we will place the Beautybox in its larger context as a smart device. In section 4, we will describe the materials and methods used in creating the Beautybox, walk through a single user session with the Beautybox, and describe the resulting state of the system. In section 5, we will describe the work currently in progress to develop an application to demonstrate the data analysis potential of this use case. In section 6, we will discuss the wider implications of this work. In section 7 we will state our position about the impact that we believe the HAT project can have.

\section{SCOPE}

In this section, we will explain the scope of this position paper and what this article is and is not about.

This article describes work in progress on the Automagic Box of Beauty, a prototype smart device to demonstrate a use case for the HAT platform.

The contribution of this article is to show a use case of the Beautybox as a motivating example for user-centered decision support, enabling individual users to capture contextual intelligence (Vermesan et al., 2014) (Dourish, 2004) about their own daily activities, as part of an ecosystem of smart devices which offer almost limitless potential for generativity (Zittrain, 2008) for the individual as well as for application developers, businesses and other stakeholders.

The Beautybox itself is a prototype, with all the limitations of a prototype, and we do not present it as a design object in its own right. We also do not claim that the Beautybox is innovative as a smart device. Smart cabinets already exist on the market, and more advanced inventory readers have been in the realm of student projects within the last five years.

The innovation is in the Beautybox's role in enabling user-centered decision support and its place in the HAT ecosystem, which we will describe in sections 5 and 6 .

\section{BACKGROUND}

In this section, we will place the Beautybox in its larger context as a smart device.

The current popularity of personal activity tracking devices such as the Fitbit proves that quantifying personal behaviour has mainstream appeal.

However, personal data requires a device to capture it $(\mathrm{Ng}, 2014)$; without the device, there is no data. This is the Beautybox's place in the HAT ecosystem.

Fitbits aside, we must first ask: what would motivate an individual user to add to their life yet another conduit through which data may flow out to destinations unknown? Why would someone ever give data away to a company? The question is rhetorical. Of course, in order to make any purchase online we have to give at least some data to a company - name, address, phone number, credit card number - but that is just the data we know we are giving away. Many things can also be inferred from metadata, which we may not be conscious of disclosing. As the Canadian Privacy Commission informs the public, "every time you add information about yourself, it's like filling in a survey... all without your knowledge, consent, or having ever been asked..." (Canadian Privacy Commission, quoted by Robin Hamman, at http://www.cybersoc.com/2007/11/video-onprivac.html\#.VFtUD0u6q0s accessed 6 November 2014).

Companies aggregate data and metadata to make inferences about us, but the insights gained are revealed only to the company, not to the individual who generated the data. Since the data is aggregated and anonymized, it ceases to be personal enough to be useful to the individual (other than the sufficiently motivated stalker). The individual receives benefits in the form of loyalty cards, but is not allowed to know what the company knows. If the data were truly owned by the individual, the individual would be issuing loyalty cards to the company, not the other way round.

Whatever its users' varying opinions about privacy, the Fitbit is so popular because - to state the seemingly obvious - the user benefits from being able to view and analyze the data that comes from it, 
and from the ability to combine it with data from other applications ( $\mathrm{Ng}, 2014)$.

How does the Beautybox compare to the Fitbit in this regard? The difference is that the data collected by the Beautybox is sent not to a corporate endpoint, but to the individual's HAT. Unlike the pseudoanonymized data that companies hold, all the data in the individual user's HAT will be personally identifiable, with the user having complete and finegrained control of which personal data they disclose and to whom they disclose it. To ensure trust, device manufacturers and companies using HAT data will require certification of their scrupulosity in maintaining the privacy of the data entrusted to them by HAT users.

\section{MATERIALS AND METHODS}

In this section, we will describe the materials and methods used in creating the Beautybox, walk through a single user session with the Beautybox, and describe the resulting state of the system.

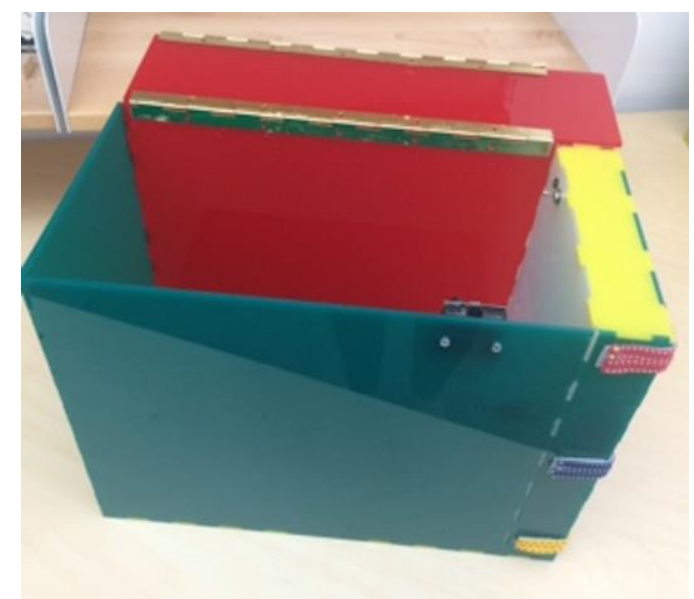

Figure 1: The Beautybox with open lid, showing the barcode scanner mounted on the front, and the assembly compartment on the right.

Figure 1 shows what the user sees when they open the Beautybox. The Beautybox is housed in a Perspex box assembly $39 \mathrm{~cm}$ long by $28.8 \mathrm{~cm}$ high by $20 \mathrm{~cm}$ wide. An interior panel divides off a $5 \mathrm{~cm}$ wide compartment at one end of the box, which houses the microcontroller and wiring assembly. On the end of the box is a removable panel held on with Velcro tape finished with strips of ribbon, enabling access to the assembly compartment.

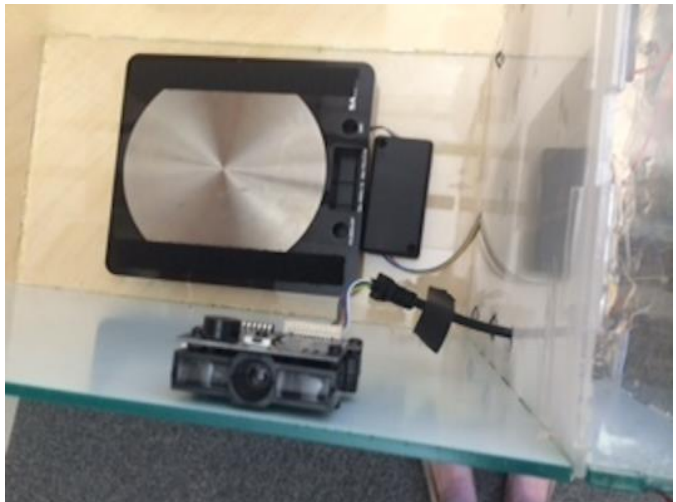

Figure 2: The barcode scanner and weighing scale mounted inside the Beautybox, with the weighing platter removed and the cables threaded through apertures in the instrument panel.

The Beautybox contains two sensors, which can be seen in Figure 2: a weighing scale on the bottom of the box (Salter 1036 "Disc" electronic kitchen scale) and a barcode scanner (Adafruit) mounted near the top edge of the front of the box. The weighing scale was adapted by the addition of a specially designed circuit board to send the weight sensor data to the Arduino Mega microcontroller.

To hide the scale and extend its weighing surface, a $33.5 \mathrm{~cm} \times 19 \mathrm{~cm}$ piece of $5 \mathrm{~mm}$ black Perspex was affixed on top with Velcro strips; this Perspex weighing platter became the floor of the box. The cables for both sensors are threaded through apertures to the other side of the instrument panel.

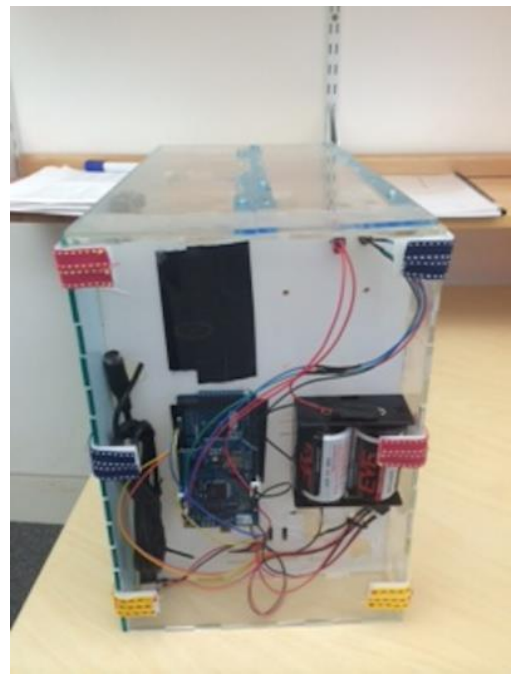

Figure 3: Beautybox with transparent end panel, showing the assembly compartment. 
The assembly compartment is shown in Figure 3. The Arduino Mega is mounted with Velcro and has an Adafruit WiFi Shield mounted on top of it to provide network connectivity and push the sensor data to the HAT endpoint. The barcode scanner's PS2 cable, as well as the load cell connectors, are wired to the Arduino, as are the LED light that displays system status to the user (Figure 4). The power is supplied by $2 \times 3.5 \mathrm{~V}$ lithium batteries (Figure 3 ) and the power supply operated by a lever switch (Figure 4).

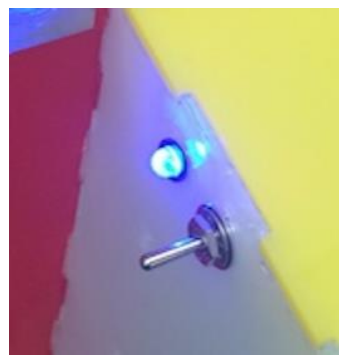

Figure 4: LED light displaying system status to the user.

\subsection{Walkthrough: using the Beautybox}

In this subsection, we will walk through a single user session with the Beautybox.

To activate the system, the user turns the power switch to ON. The LED light turns red as the system initializes.

The LED light turns blue as the WiFi Shield connects to the user's wireless network.

The LED light turns red as the system initializes the weighing scale and records the starting weight of the box contents.

The LED light turns green when the barcode scanner becomes available.

The user takes an item out of the box and scans the barcode. The LED light turns red while the system polls for a change in weight readings. Weight is calculated by a moving average over 250 readings taken at 100-millisecond intervals.

If the system times out before a weight change is detected, the LED turns green and the barcode scanner becomes available again. Otherwise, if a weight change is detected, the LED turns blue and the system uses the HAT REST API to send the barcode and item weight to the HAT endpoint. Item weight is the absolute difference between the latest and previous weights of the box contents.

When the database transaction is complete, the LED turns green to indicate that the barcode scanner is available.

When the session has finished, the user manually powers off the system.

\subsubsection{Resulting State of the System}

In this subsection, we will describe the resulting state of the system.

The DP0's HAT now contains two new items of sensor data: the timestamped barcode identifying the product used, and the timestamped item weight.

The simplest analysis that the user can do with this sensor data is to track the rate of consumption of each product. As the product - for example shampoo - is taken out, used, and put back into the Beautybox, the item weight will go down, and then suddenly go up when the product is replaced.

\section{WORK IN PROGRESS}

In this section, we will describe the work currently in progress to develop an application to demonstrate the data analysis potential of this use case.

We are currently developing a simple application to demonstrate a use case of analysis of the Beautybox data. Using the combined barcodes, timestamps, and item weights, the average daily usage of a product is simple to calculate, and the system can predict the amount of time before a product will need to be replaced.

With this information, we will implement automated reordering functionality - like a subscription service, but more efficient and less expensive because the reorder will be placed just-intime rather than at regular intervals.

Not everyone wishes to use the same shampoo every time, and products sometimes become unavailable. In this situation, a use case for decision support becomes apparent. Using the barcode, it is possible to search publicly available APIs such as the Tesco API and find product information; the search criteria depend on the user's reasons for selecting that particular shampoo in preference to others.

Supposing a user suffers from scalp problems and must use a very specific treatment shampoo. The shampoo is expensive, difficult to find, and subject to withdrawal by the manufacturer at unpredictable intervals. The user might know of a list of acceptable alternative products and specify the product names. But what if the user needs help to identify an alternative product? In that case, the system can search for a shampoo that contains the required active ingredients (coal tar, salicylic acid) or without the undesired ingredients (proteins, silicones).

Not every user has such specific preferences. With the finished application, users will be able to specify how important it is for them to replace the 
Beautybox items with the exact product each time. Perhaps a user will accept any shampoo as a substitute, or wants to receive a random shampoo (possibly within a certain price range) each time.

Chen et al. (Chen et al., 2013) define the usage context of a product as "all aspects describing the context of product use that vary under different use conditions and affect product performance and/or customer preferences for the product attributes". Taking the application a step further, we will work with each DP0 to contextualize the Beautybox data according to their respective preferences and needs (Dourish, 2004). For example, imagine the box contains a hair-straightening product and the user runs out of it before the system has a chance to reorder it. Why has this happened? On examination, the straightening product shows a very low daily usage rate until there is a sudden spike in consumption. Imagine this user has also been recording weather data in the HAT, making it possible to combine it with the Beautybox data. Looking at the combined weather and Beautybox sensor data, it turns out that the straightening product was only used on rainy days with a chance of thunderstorms, but otherwise never touched. We could therefore add functionality to the application by contextualizing product usage with weather, anticipating increased usage of the straightening product for the week ahead if thunderstorms are predicted.

\section{DISCUSSION}

In this section, we will discuss the wider implications of this work.

Because the variety of user preferences for contextualization is potentially limitless, the average user is unlikely to have the resources to perform all their desired analysis on their own, and so the role of application developers within the HAT ecosystem becomes apparent.

This brings us to the topic of generativity. Zittrain (Zittrain, 2008) defines generativity as "a system's capacity to produce unanticipated change through unfiltered contributions from broad and varied audiences." Zittrain identified five features of a solution conforming to the generative pattern: leverage (making a difficult job easier), adaptability (alterable for a variety of purposes), ease of mastery (requiring minimal training to use and extend), accessibility (ease of obtaining and developing a working system), and transferability (ease of distributing updates).

The HAT platform, even at the prototype stage, has nearly all of Zittrain's features of a generative solution. It has the leverage of its "human-thinkalike" (Ng, 2014) database model, as well as the adaptability of the REST API which provides interoperability to any HAT-ready device and consequent combination of device data for decision support. The database model and REST API combine to provide ease of mastery by making it simple to make a device HAT-ready and simple to combine data downloaded from the HAT; the platform's openness provides the requisite accessibility. Only transferability remains unaddressed at the prototype stage, in that changes affecting the Beautybox must be manually uploaded to the microprocessor. Improving transferability will be addressed in future work.

Businesses and product designers also stand to gain unprecedented insights from the purchase of data that has been contextualized and horizontally integrated according to preferences defined by the users themselves. As Chen et al. (Chen et al., 2013) explain, "The usage context may also have a significant impact on the product performance, which is not considered in existing methods that simply treat product performance as 'constant' across all customers and usage contexts in choice modeling."

\section{CONCLUSIONS}

In this position paper, we have described the Automagic Box of Beauty, a prototype smart device to demonstrate an example use case for usercentered decision support within the Hub-of-allThings, a personal data store for the Internet of Things that places ownership of personal data in the hands of the individual $(\mathrm{Ng}, 2014)$. In section 1 , we explained what the HAT project is, what the Beautybox is, what a DP0 is and the current stage of deployment of the Beautybox, and how we intend to use the data generated by the Beautybox. In section 2 , we explained the scope of this position paper and what this article is and is not about. In section 3, we placed the Beautybox in its larger context as a smart device. In section 4, we described the materials and methods used in creating the Beautybox, walked through a single user session with the Beautybox, and described the resulting state of the system. In section 5, we described the work currently in progress to develop an application to demonstrate the data analysis potential of this use case. In section 6 , we discussed the wider implications of this work. In this section, we will state our position about the impact that we believe the HAT project can have.

The use case described in this article is just one example out of a potentially limitless set of 
scenarios in which the HAT can facilitate decision support for a vast variety of individual user preferences.

By returning personal data to the control of the individual, and contextualizing raw data that until now has been held in vertical silos $(\mathrm{Ng}, 2014)$, users, with the help of application developers providing for their needs, will have an opportunity for the kind of decision support that was previously only available at the enterprise level.

The individual user owns all their own data, can see all their own data, is uniquely capable of interpreting what the data tells them about their own behaviour patterns, and will stimulate a market for application developers to provide decision support tools at the individual user level and for businesses to gain unprecedented insight into usage context.

\section{ACKNOWLEDGEMENTS}

The custom circuit board for the weighing scale and the design of the Perspex box structure, as well as copious and invaluable expert advice, were provided by Brian Jones.

The work, and in particular the wiring of the small components, could not have been completed without the assistance and advice of Andrius Aučinas and Carlos Molina Jiménez.

\section{REFERENCES}

Chen, W., Hoyle, C., Wassenaar, H.J., 2013. A choice modeling approach for usage context-based design. In Decision-Based Design, Springer. London, pp.255285.

Dourish, P., 2004. What we talk about when we talk about context. Personal and Ubiquitous Computing 8(1), pp.19-30.

$\mathrm{Ng}$, I., 2014. Engineering a market for personal data: the Hub-of-all-Things. RCUK Digital Economy HAT Project. Available online at http://hubofallthings.com/hat-briefing-paper/ Accessed 6 November 2014.

Vermesan, O., Friess, P., Guillemin, P., Sundmaeker, H., Eisenhauer, M., Moessner, K., Arndt, M., Spirito, M., Medagliani, P., Giaffreda, R., Gusmeroli, S., Ladid, L., Serrano, M., Hauswirth, M., Baldini, G., 2014. Internet of Things strategic research and innovation agenda. In Internet of Things - from research and innovation to market deployment, River Publishers. Aalborg, pp.7-142. Vermesan, O., Friess, P. (Eds.).

World Economic Forum, 2013. Unlocking the value of personal data: from collection to usage.
Zittrain, J., 2008. The future of the Internet - and how to stop it, Yale University Press. New Haven.

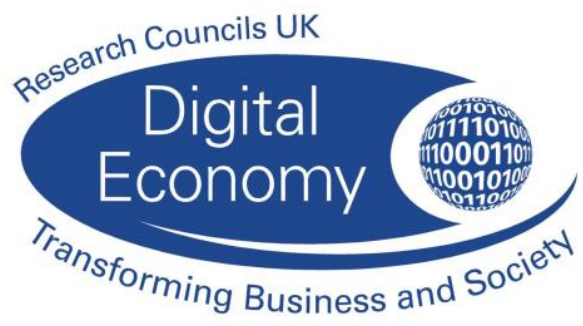

The HAT project is funded by the RCUK Digital Economy Programme. 